\title{
Evaluación del impacto clínico y económico del paciente con diabetes mellitus con acceso a automonitoreo en Panamá
}

\section{Evaluation of the clinical and economic impact of patient management with diabetes mellitus with access to self-monitoring in Panama}

\author{
Guadalupe Pérez ${ }^{1}$, Ycly Jaén-Fernández², Ricardo Correa ${ }^{3}$ y Javier Picó $4 *$
}

${ }^{1}$ Endocrinología; ${ }^{2}$ Medicina Familiar. Caja del Seguro Social, Ciudad de Panamá, Panamá; ${ }^{3}$ Diversidad para la educacion médica, University of Arizona College of Medicine, Phoenix, Estados Unidos; ${ }^{4}$ Consultoría de negocios, LifeSciences Consultants, Ciudad de México, México

\section{RESUMEN}

Entre el 2014 y el 2017 se implementó en la Caja del Seguro de Social de Panamá, en 32 puntos de contacto, un programa de automonitoreo que otorgaba a pacientes con diabetes mellitus: glucómetro, lancetas, tiras reactivas, software de descarga y análisis de mediciones de glucosa en sangre y servicios de nutrición para gestionar la diabetes. Como objetivo principal, este trabajo evalúa el impacto clínico del programa de automonitoreo por medio de la reducción de la glucosa en sangre (al no contar con la medición de hemoglobina glucosilada [HbA1c] protocolizada durante el programa). Como objetivo secundario evalúa el impacto económico, dado el cambio en muertes y complicaciones asociado a esta intervención, y lo proyecta en el tiempo según la probabilidad de dichos eventos. Como resultado del análisis, se observó un aumento del $6 \%(\mathrm{p}<0.000)$ en la tasa de pacientes bajo control glucémico ( $\mathrm{HbA} 1 \mathrm{c}<7 \%$ ). Adicionalmente, se observó un impacto positivo en los niveles de glucosa en el $82 \%$ de los pacientes. Se estimó un ahorro de 20.9 millones de dólares en relación con los pacientes que participaron en la intervención y que contaban con la información suficiente para el análisis. Nuestros resultados motivan la discusión del impacto clínico y económico del automonitoreo.

Palabras clave: Diabetes mellitus. Costos y análisis de costos. Registros. Panamá. Automonitoreo de glucosa en sangre.

\begin{abstract}
Between 2014 and 2017, Caja de Seguridad Social (CSS) in Panama implemented in 32 contact points a self-monitoring program that provided patients with diabetes mellitus a glucometer, lancets, test strips, software for downloading and analyzing the blood glucose levels, and nutrition services for the diabetes management of the patients. The main objective of this study is to assess the clinical impact of the self-monitoring program, through the reduction of the blood glucose (there was not a protocol to measure glycated haemoglobin [HbA1c] during the program). As a secondary objective, assess the economic impact due to the change in deaths and complications associated with this intervention and project them through time given these events probability. As a result of the analysis, a $6 \%$ increase $(p<0.000)$ was observed in the rate of patients under glycemic control ( $\mathrm{HbA} 1 \mathrm{C}<7 \%)$. Also, a positive impact on glucose levels was observed in $82 \%$ of patients. Savings of 20.9 million dollars were estimated for the patients that participated in the program and had the required information for the analysis. Our results motivate the discussion of the clinical and economic impact of self-monitoring.
\end{abstract}

Key words: Diabetes mellitus. Costs and costs analysis. Registries. Panama. Blood glucose self-monitoring.

\section{Correspondencia:}

* Javier Picó

E-mail: jpicolaconsultingls.com
Fecha de recepción: 28-08-2020

Fecha de aceptación: 04-02-2021

DOI: 10.24875/ALAD.20000026
Disponible en internet: 17-03-2021 Rev ALAD. 2021;11:29-39

2248-6518 / @ 2021 Asociación Latinoamericana de Diabetes. Publicado por Permanyer. Este es un artículo open access bajo la licencia CC BY-NC-ND (http://creativecommons.org/licenses/by-nc-nd/4.0/). 


\section{INTRODUCCIÓN}

La diabetes mellitus (DM) es una enfermedad caracterizada por un alto nivel de glucosa resultado de defectos en la capacidad del cuerpo para producir o utilizar insulina ${ }^{1}$ y es actualmente reconocida mundialmente como una epidemia debido a su creciente prevalencia ${ }^{2}$. La diabetes está asociada con varias complicaciones, como: accidente cerebrovascular, nefropatía, infarto de miocardio, amputación, cataratas, glaucoma, retinopatía, neuropatía, úlceras, coma y muerte. Las comorbilidades y la mortalidad relacionadas con la DM representan un gasto de cientos de millones de dólares al año, proyectado a aumentar en los siguientes años 3,4 .

En la literatura se ha establecido una relación entre los niveles de glucosa y la incidencia de complicaciones relacionadas con la diabetes ${ }^{5,6}$, por lo que mantener niveles controlados de glucosa es esencial para controlar el gasto asociado a la diabetes. Según la Asociación Latinoamericana de la Diabetes $(A L A D)$ «El automonitoreo de la glucosa se ha convertido en uno de los elementos fundamentales en el cuidado y el control de los pacientes con diabetes mellitus» ${ }^{7}$, estableciendo el autocuidado como la medición frecuente de glucosa de los pacientes como prioritario para el control de la enfermedad. Varios estudios ${ }^{8-10}$, además, han podido demostrar la relación entre el alza en el nivel de glucosa y la incidencia de complicaciones, lo que resalta la importancia de mantener dichos niveles bajo control.

En la actualidad, las guías clínicas internacionales definen como meta de tratamiento mantener un nivel de hemoglobina glucosilada (HbA1c) menor al 7\%11-13, lo cual está asociado con el control glucémico; sin embargo, esta meta puede ser individualizada dependiendo de factores como riesgo individual de hipoglucemia, duración de la enfermedad, comorbilidades, complicaciones vasculares, preferencia del paciente y recursos disponibles ${ }^{11}$. Se ha demostrado que con niveles de $\mathrm{HbA} 1 \mathrm{c}$ inferiores al $7 \%$ se reducen las complicaciones relacionadas con la diabetes ${ }^{14,15}$. Asimismo, la implementación de programas de educación y de medición de niveles pueden ayudar a los pacientes a alcanzar o mantenerse por debajo de esta meta ${ }^{16}$.

La ADA estima que dichas intervenciones pueden generar reducciones en los niveles de $\mathrm{HbA} 1 \mathrm{c}$ del $1 \% 11$, y que su implementación resulta costo-efectiva para los sistemas de salud. Menciona también que los programas de automonitoreo deben estar integrados en planes integrales de automanejo de la enfermedad $^{6}$. Asimismo, una revisión sistemática realizada por Liang, et al. en el $2011^{17}$ mostró que los programas de automonitoreo y educación pueden generar una reducción en los niveles de $\mathrm{HbA} 1 \mathrm{c}$ en pacientes con diabetes, en promedio de un $0.8 \%$ específicamente en pacientes con diabetes tipo 2 .

En Panamá se estima una prevalencia de DM de 375 mil pacientes, representando al 9\% de la población ${ }^{18,19}$. En un estudio realizado por Barcelo, et al. en el 2015 se estimaron los costos directos e indirectos de la diabetes en Panamá y en México ${ }^{3}$. El costo directo se estimó en 364 millones de dólares anuales. Sin embargo, un estudio de prevalencia de factores de riesgo asociados a enfermedad cardiovascular (PREFREC) realizado en Panamá mostró que solo el 59.9\% de los pacientes con diagnóstico de DM se han realizado una prueba de $\mathrm{HbA1c}$ en su vida ${ }^{20}$.

En Panamá, la Caja de Seguridad de Social (CSS) es la Institución autónoma encargada de administrar prestaciones a favor de los trabajadores públicos y privados, con cobertura del $75 \%$ de la población ${ }^{21}$.

Entre el 2014 y el 2017, adicional a la atención médica, el tratamiento general y el uso de fármacos prescritos, se implementó en la CSS de Panamá un 
programa de automonitoreo en el cual se otorgaba a pacientes con DM un glucómetro, lancetas y las tiras reactivas correspondientes a su esquema de tratamiento, de manera gratuita, así como soporte por parte de navegadoras de salud (nutricionistas educadoras en diabetes).

Es relevante demostrar que programas como el de la CSS en Panamá, a pesar de requerir una inversión inicial considerable, pueden ayudar a mejorar la vida de los pacientes, mediante el empoderamiento en el manejo de su enfermedad, reduciendo el gasto producido por las complicaciones asociadas al descontrol glucémico.

Con este estudio buscamos como objetivo principal estimar el impacto clínico de la intervención sobre el control glucémico de los pacientes de la CSS de Panamá en sus pacientes con diabetes que se implementó del año 2014 hasta el año 2017.

Como objetivo secundario se analizó el impacto económico en la CSS de Panamá dada la disminución en muertes y complicaciones asociadas a la intervención.

\section{MATERIAL Y MÉTODOS}

El presente trabajo es un estudio transversal retrospectivo de series de tiempo interrumpido. La información fue recopilada de 35 mil pacientes invitados a formar parte de un programa de automonitoreo en el cual se les otorgó un glucómetro, lancetas y tiras reactivas para el manejo de su diabetes.

Durante el programa se contempló la compra anual de 31.8 millones de tiras, lancetas y otros accesorios. Además, como parte del programa se impartieron capacitaciones de hasta 24 horas a médicos y a profesionales de la salud para el diagnóstico y manejo óptimo de la diabetes. Los 32 centros de la CSS participantes contaron con la presencia de navegadoras de salud (nutricionistas educadoras en diabetes). Ellas se encargaron de proveer por lo menos una asesoría a cada paciente en cuanto a hábitos alimenticios y de ejercicio, así como de enseñarles a usar e interpretar el glucómetro. Las navegadoras de salud también fueron encargadas de recabar los datos de los registros de niveles de glucosa guardados en los glucómetros de los pacientes con el limitante de que no tenían acceso a los expedientes clínicos de los pacientes. Los pacientes tenían la responsabilidad de asistir periódicamente a la clínica para que las navegadoras de salud pudieran guardar los registros en una base de datos y de medir sus niveles de glucosa periódicamente conforme a lo recomendado; por medio de un número identificador de paciente (ID), las navegadoras ligaban a su base de datos dichos registros. Además, se les dio a los pacientes un programa de educación en relación con la diabetes, su tratamiento y prevención. Al final del programa, se obtuvieron más de 12 millones de registros guardados de entre 2014-2017. Los datos confidenciales de los pacientes no fueron publicados o entregados a personas sin autorización. Estos datos fueron procesados para obtener el promedio trimestral de los niveles de glucosa, el cual se tradujo a niveles de HbA1c mediante una calculadora de equivalencias ${ }^{22}$. Llamaremos intervención al programa de automonitoreo: educación nutricional y de estilo de vida, implementación de glucómetro, sumado al uso de fármacos y la atención médica provista por los médicos de la CSS.

Inicialmente, el proyecto de la CSS no buscaba analizar el impacto clínico y económico de la intervención, por lo cual no hubo un registro sistemático y/u obligatorio de la glucosa en sangre de los pacientes descargada automáticamente de los glucómetros entregados y de los niveles de HbA1c de los pacientes que su médico tratante solicitaba al laboratorio 
clínico y registraba en el expediente de la CSS, al cual no se tuvo acceso. Para solucionar las inconsistencias en el manejo de la información y obtener resultados confiables, los niveles de glucosa fueron procesados para obtener el promedio trimestral de los niveles de glucosa, el cual se tradujo a niveles de HbA1c mediante una calculadora de equivalencias. También se utilizó solamente la información de los pacientes con más de un año en el programa, debido a que un periodo de menos de un año no contaba con los datos suficientes por paciente para eliminar los picos de glucosa en sangre derivados del comportamiento del paciente. Cada paciente registró 9.8 mediciones promedio de glucosa en sangre, con una desviación estándar de 3.6, número mayor de mediciones de glucosa en sangre que las utilizadas en artículos publicados donde se ha concluido que los valores calculados de $\mathrm{HbA1c}$ a partir de los datos de la glucosa en sangre coincidieron en general con los valores de $\mathrm{HbA} 1 \mathrm{C}$ medidos ${ }^{23}$.

Se definieron dos criterios de inclusión: pacientes que cumplieron al menos un año en el programa y pacientes que presentaron récord médico completo (definido como aquel que cuenta con tipo de diabetes y sexo). Se definieron dos criterios de exclusión: pacientes que no contaban con al menos una medición de glucosa registrada y pacientes con menos de un año de diferencia entre la primera y la última medición de glucosa registradas.

\section{ANÁLISIS ESTADÍSTICOS}

Para obtener un mejor análisis con la información disponible, se definieron criterios de inclusión y exclusión distintos para cada análisis propuesto. Para estimar el impacto clínico de la intervención se consideraron los dos criterios de exclusión, resultando en 15.2 mil pacientes analizados. Por otro lado, para aproximar el impacto económico se consideró el primer criterio de exclusión, resultando en 19.4 mil pacientes (Fig. 1).

Definimos como significativos los resultados con valor $\mathrm{p}<0.05 \mathrm{y}$ el control glucémico como valores inferiores al $7 \%$ de HbA1c. El análisis de valores $\mathrm{p}$ se realizó mediante la prueba $\mathrm{t}$ de Student pareada. Así como la prueba $\chi^{2}$ para probar dependencia de variables, con diferencia de proporciones se contrastó el porcentaje de pacientes con mejora en la intervención entre hombres y mujeres (Tablas 1-4).

El impacto clínico del programa fue estimado al comparar la distribución inicial y final de la HbA1c con base en los pacientes con al menos un año de seguimiento.

Para estimar el impacto económico de la intervención, se consideraron los costos de las complicaciones asociadas a la enfermedad. Se comparó el número de complicaciones y muertes esperadas en la población que participó en la intervención con las esperadas en la misma población si su enfermedad hubiese progresado naturalmente. Esta última se simuló a partir de las mediciones observadas en los pacientes por año y asumiendo un alza en $\mathrm{HbA} 1 \mathrm{c}$ del $0.2 \%{ }^{24}$, ponderándolas cada año con las nuevas observaciones.

El impacto económico fue calculado como la diferencia del gasto en complicaciones ${ }^{5,6}$. Dada la diferencia de distribución de HbA1c que mostraron los participantes de la intervención, frente a la tendencia en complicaciones de la población de la CSS sin intervención, siguiendo la evolución de la DM (nivel de complicación y HbA1c en el tiempo). Este impacto fue medido sobre una muestra de 19.4 mil pacientes que contaron con al menos una medición registrada de glucosa y los resultados reportados fueron extrapolados al universo de pacientes. Los costos utilizados para estimar el impacto económico fueron 


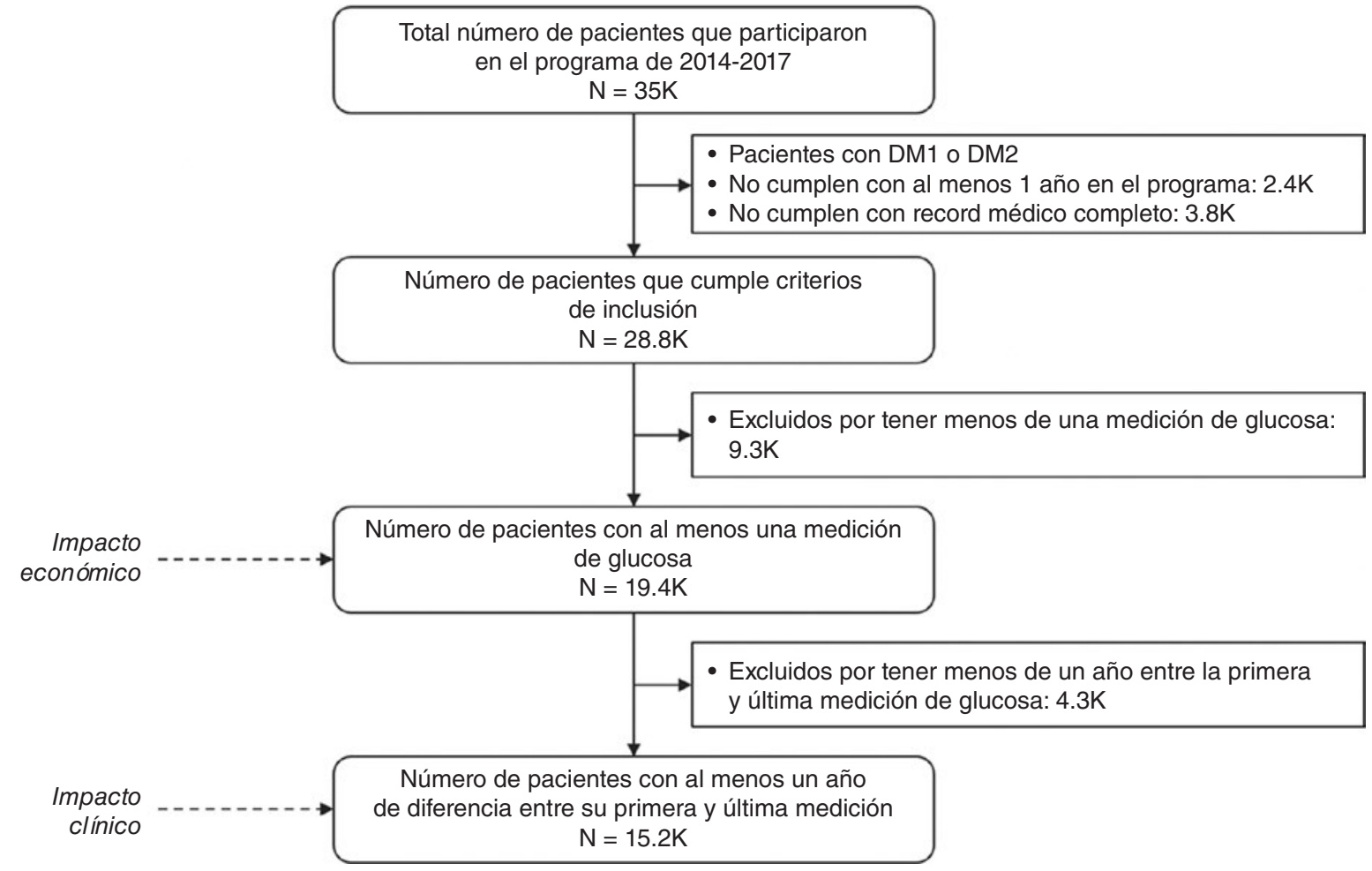

Figura 1. Número de pacientes analizados y criterios de inclusión para medir el impacto económico y clínico de la intervención. DM: diabetes mellitus.

Tabla 1. Pruebas de significancia de variables clínicas. Pruebas estadísticas del cambio en niveles de hemoglobina glucosilada (HbA1c)

\begin{tabular}{|c|c|c|c|c|}
\hline & Resultado inicial & Resultado final & Cambio & Valor $\mathrm{p}^{*}$ \\
\hline Población con niveles de $\mathrm{HbA} 1 \mathrm{c}<7 \%$ & $65 \%$ & $71 \%$ & $6 \%$ & $<0.000$ \\
\hline $\begin{array}{l}\text { Nivel promedio de } \mathrm{HbA1c} \text { de los pacientes que redujeron } \\
\text { sus niveles entre su primera y última medición }\end{array}$ & $7.4 \%$ & $6.1 \%$ & $1.3 \%$ & $<0.000$ \\
\hline
\end{tabular}

*Estimado con la prueba $t$ de Student pareada.

TABLA 2. Pruebas de significancia de variables clínicas. Prueba estadística de proporción de hombres/mujeres por debajo del punto de corte de hemoglobina glucosilada (HbA1c)

\begin{tabular}{|l|c|c|c|}
\hline $\begin{array}{l}\text { Proporción de hombres } \\
\text { y mujeres con niveles } \\
\text { de HbA1c }<7 \%\end{array}$ & $\begin{array}{c}\text { Hombres } \\
(32 \%)\end{array}$ & $\begin{array}{c}\text { Mujeres } \\
(68 \%)\end{array}$ & Valor * $^{*}$ \\
\hline Resultado inicial & $33.3 \%$ & $66.7 \%$ & $<0.000$ \\
\hline Resultado final & $32.9 \%$ & $67.1 \%$ & $<0.000$ \\
\hline
\end{tabular}

*Estimado con la prueba $\chi^{2}$. compartidos por la institución. En caso de que algún costo no estuviera disponible, se estimó a partir de los costos de otras instituciones de salud pública similares. Específicamente, los costos unitarios de las complicaciones se estimaron basados en los Grupos Relacionados al Diagnóstico del IMSS ${ }^{25}$ y se transformaron a un valor aplicable en Panamá por medio de la diferencia del gasto per cápita de la diabetes en México y Panamá3. Todos los costos fueron revisados y validados por la CSS (Tabla 5). 
TABla 3. Pruebas de significancia de variables clínicas. Análisis de dependencia entre tipo de diabetes mellitus (DM) y resultados glucémicos

\begin{tabular}{|c|c|c|c|c|}
\hline Nivel de control glucémico entre tipos de diabetes & DM1 (4\%) & DM2 (96\%) & Total (100\%) & Valor $\mathrm{p}^{*}$ \\
\hline Resultado inicial & $46 \%$ & $66 \%$ & $65 \%$ & $<0.000$ \\
\hline Resultado final & $47 \%$ & $73 \%$ & $72 \%$ & $<0.000$ \\
\hline
\end{tabular}

*Estimado con la prueba $\chi^{2}$.

Tabla 4. Pruebas de significancia de variables clínicas. Prueba de diferencia de proporciones para porcentaje de respuesta en hombres y mujeres

\begin{tabular}{|l|c|c|c|c|c|}
\hline & $\begin{array}{c}\text { Resultado } \\
\text { inicial }\end{array}$ & $\begin{array}{c}\text { Resultado } \\
\text { final }\end{array}$ & Cambio & $\begin{array}{c}\text { Estadístico } \\
\text { de prueba }\end{array}$ & \begin{tabular}{c} 
Prueba \\
\hline de hombres con niveles de HbA1c $<7 \%$
\end{tabular} \\
\hline \% de mujeres con niveles de HbA1c $<7 \%$ & $68 \%$ & $74 \%$ & $-5.4 \%$ & $\begin{array}{c}\text { Diferencia de proporciones } \\
(z=2.43, p=0.007)\end{array}$ \\
\hline
\end{tabular}

HbA1c: hemoglobina glucosilada.

TABLA 5. Probabilidad anual de sufrir una complicación relacionada con la diabetes por nivel de hemoglobina glucosilada ( $\mathrm{HbA1c}$ ) y costo estimado de la intervención

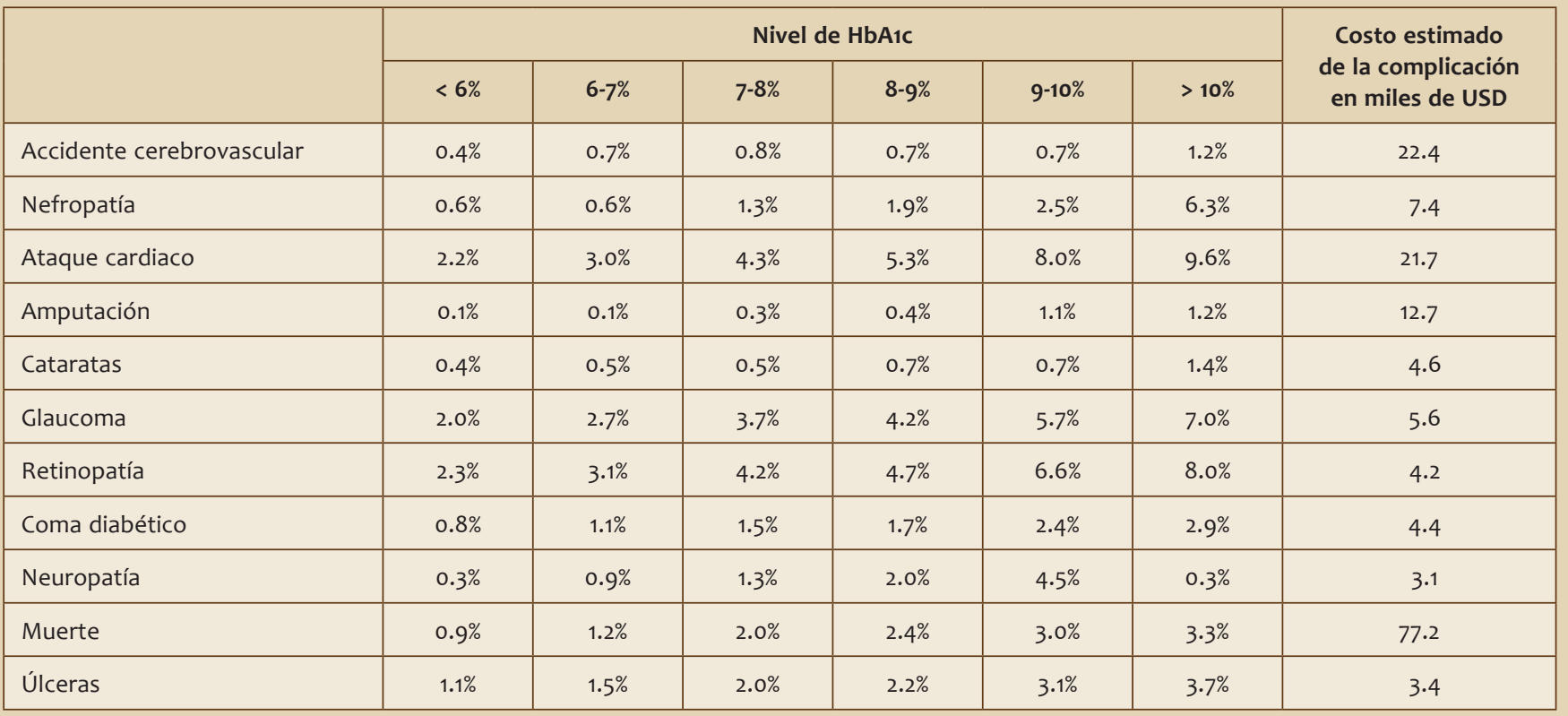

Adaptada de Stratton, et al., 20005, Barraza-Lloréns, et al., $2013^{6}$ y Roche²3.

\section{RESULTADOS}

La tabla 6 muestra la información de tipo de diabetes y sexo de los pacientes; además del número de pacientes en control glucémico, con la primera medición registrada de cada paciente y la última. De entre los pacientes que registraron más de una medición de nivel glucémico únicamente se contó con 
TABLA 6. Distribución de los pacientes considerados para el análisis principal

\begin{tabular}{|c|c|c|c|c|c|}
\hline \multirow[t]{2}{*}{ Inicial } & \multicolumn{2}{|c|}{ Hombres } & \multicolumn{2}{|c|}{ Mujeres } & \multirow[t]{2}{*}{ Total } \\
\hline & DM1 & $\mathrm{DM}_{2}$ & DM1 & DM2 & \\
\hline $\mathrm{HbA}_{1} \mathrm{C}<7 \% \mathrm{n}(\%)$ & $116(46 \%)$ & $3,188(69 \%)$ & $183(45 \%)$ & $6,428(65 \%)$ & $9,915(65 \%)$ \\
\hline $\mathrm{HbA}_{1} \mathrm{C}>7 \% \mathrm{n}(\%)$ & $134(54 \%)$ & $1,408(31 \%)$ & $220(55 \%)$ & $3,478(35 \%)$ & $5,240(35 \%)$ \\
\hline Total & 250 & 4,596 & 403 & 9,906 & 15,155 \\
\hline \multirow[t]{2}{*}{ Final } & \multicolumn{2}{|c|}{ Hombres } & \multicolumn{2}{|c|}{ Mujeres } & \multirow[t]{2}{*}{ Total } \\
\hline & DM1 & $\mathrm{DM}_{2}$ & DM1 & DM2 & \\
\hline $\mathrm{HbA}_{1} \mathrm{C}<7 \% \mathrm{n}(\%)$ & $113(45 \%)$ & $3,454(75 \%)$ & $191(47 \%)$ & $7,083(72 \%)$ & $10,841(72 \%)$ \\
\hline $\mathrm{HbA}_{1} \mathrm{C}>7 \% \mathrm{n}(\%)$ & $137(55 \%)$ & $1,142(25 \%)$ & $212(53 \%)$ & $2,823(28 \%)$ & $4,314(28 \%)$ \\
\hline Total & 250 & 4,596 & 403 & 9,906 & 15,155 \\
\hline
\end{tabular}

DM: diabetes mellitus; HbA1C: hemoglobina glucosilada.

la información de edad de 6.1 mil pacientes, teniendo como media 61.2 años de edad, con una desviación estándar de 12.3 años, el resto de los pacientes no contaron con registro de edad.

\section{IMPACTO CLÍNICO DE LA INTERVENCIÓN}

De los 15.2 mil pacientes con al menos un año de diferencia entre su primer y última medición guardada de glucosa, el $82 \%$ se mantuvieron en control glucémico o redujeron sus niveles de HbA1c (Fig. 2), cuando se comparó el primer nivel calculado de HbA1c con el último. Se observó un aumento del $6 \%$ $(p<0.000)$ en la tasa de pacientes en control glucémico ( $\mathrm{HbA} 1 \mathrm{c}<7 \%)$. La reducción promedio absoluta de $\mathrm{HbA} 1 \mathrm{c}$ para los pacientes que redujeron sus niveles entre su primera y última medición fue de del 1.3\% (Tabla 1).

Hay una diferencia significativa $(p<0.000)$ entre la proporción de hombres y mujeres por debajo del punto de corte, antes y después de la intervención (Tabla 2). El 96\% de los pacientes tuvo DM tipo 2. Se observó dependencia entre el tipo de diabetes y el

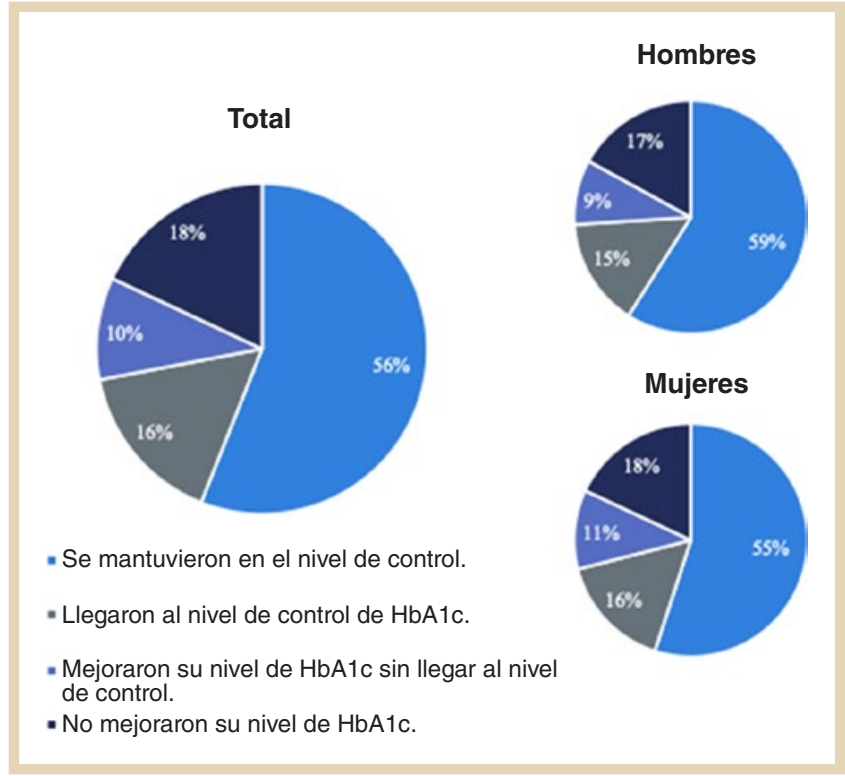

Figura 2. Distribución de impacto en los niveles de hemoglobina glucosilada ( $\mathrm{HbA1c}$ ) resultante de la intervención.

control glucémico en los pacientes antes y después de la intervención $(\mathrm{p}<0.000)$ (Tabla 3).

La prueba de diferencia de proporciones, donde el 5.4\% de los hombres y el $6.4 \%$ de mujeres pasaron a control glucémico en el último registro de niveles de glucosa, mostró diferencia significativa $(z=2.43 ; \mathrm{p}=0.007) \mathrm{y}$ mayor en las mujeres participantes (Tabla 4). 


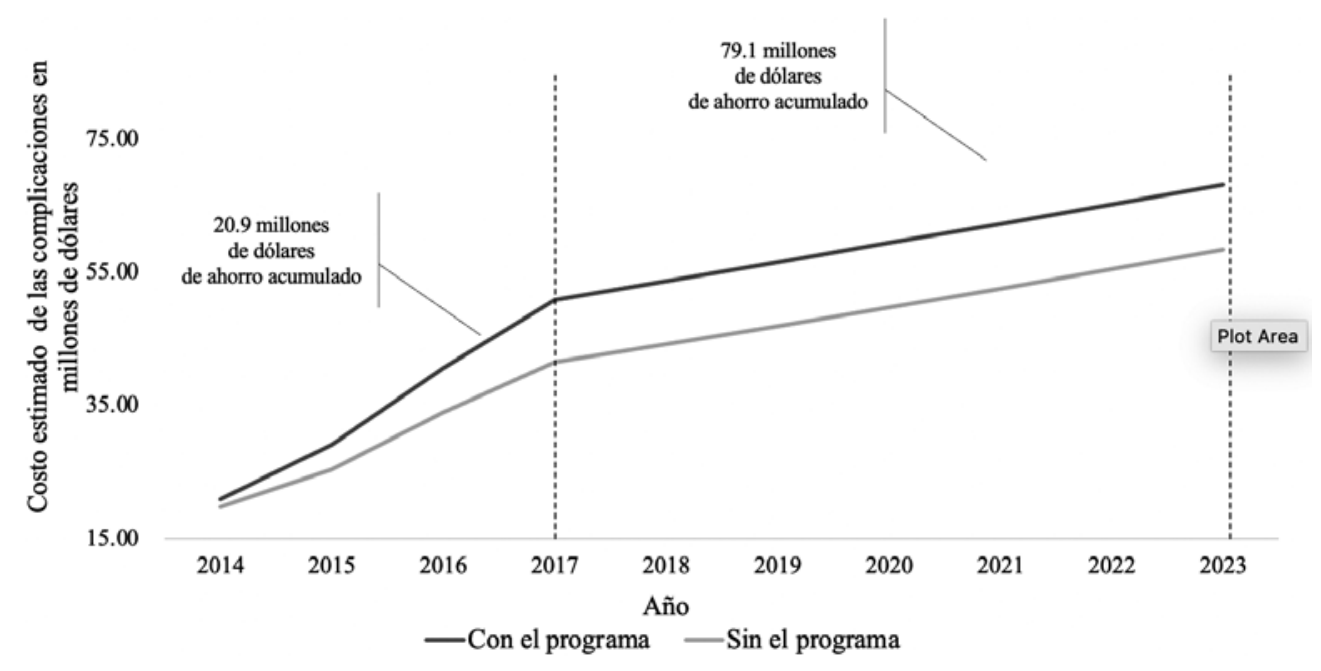

Figura 3. Costo estimado de las complicaciones por año y número de pacientes activos, extrapolado al 2023. Considerando a 19.4 mil pacientes y su año de entrada al programa.

Se identificó que las mujeres con diabetes tipo 2 fueron el tipo de paciente que lograron un mayor impacto positivo en sus niveles de HbA1c.

\section{IMPACTO ECONÓMICO DE LA INTERVENCIÓN}

El costo resultante real del programa de automonitoreo durante los cuatro años fue de 15.5 millones de dólares; siendo de 211.5 USD por paciente por año, considerando a los 35 mil pacientes y su permanencia individual en el programa.

Para el 2017, con base en las probabilidades dado el nivel de HbA1c (Tabla 5) de los pacientes, se estimó que el costo de tratar las complicaciones asociadas de los 19.4 mil pacientes con la información requerida en la intervención ascendería a 51 millones de USD, considerando la progresión natural de la enfermedad, como se muestra en la figura 3 en el rango 2014-2017.

Se calculó que en este mismo periodo la reducción asociada a la intervención en las complicaciones y muertes fue en promedio del $14.5 \%$, contra lo esperado con la progresión natural. Esta reducción asociada a la intervención pudo haber evitado alrededor de 194 muertes y 2,205 complicaciones relacionadas con la enfermedad. Al extrapolar el comportamiento de pacientes con mediciones de glucosa al total de pacientes en la intervención, la reducción en complicaciones representa un ahorro para la CSS de aproximadamente 20.9 millones de dólares (Fig. 3).

El impacto de la intervención resulta más evidente cuando se analiza la diferencia en el costo anual de las complicaciones por paciente con la distribución de HbA1c obtenida de los pacientes en el PA y de los pacientes sin el PA. En este análisis se estima que entre el 2014 y el 2017 se generaron ahorros acumulados de aproximadamente 920 dólares por paciente. (Fig. 4 y Tabla 7).

\section{DISCUSIÓN}

Se observó un aumento del 6\% $(\mathrm{p}<0.000)$ en la tasa de pacientes por debajo del punto de corte definido 


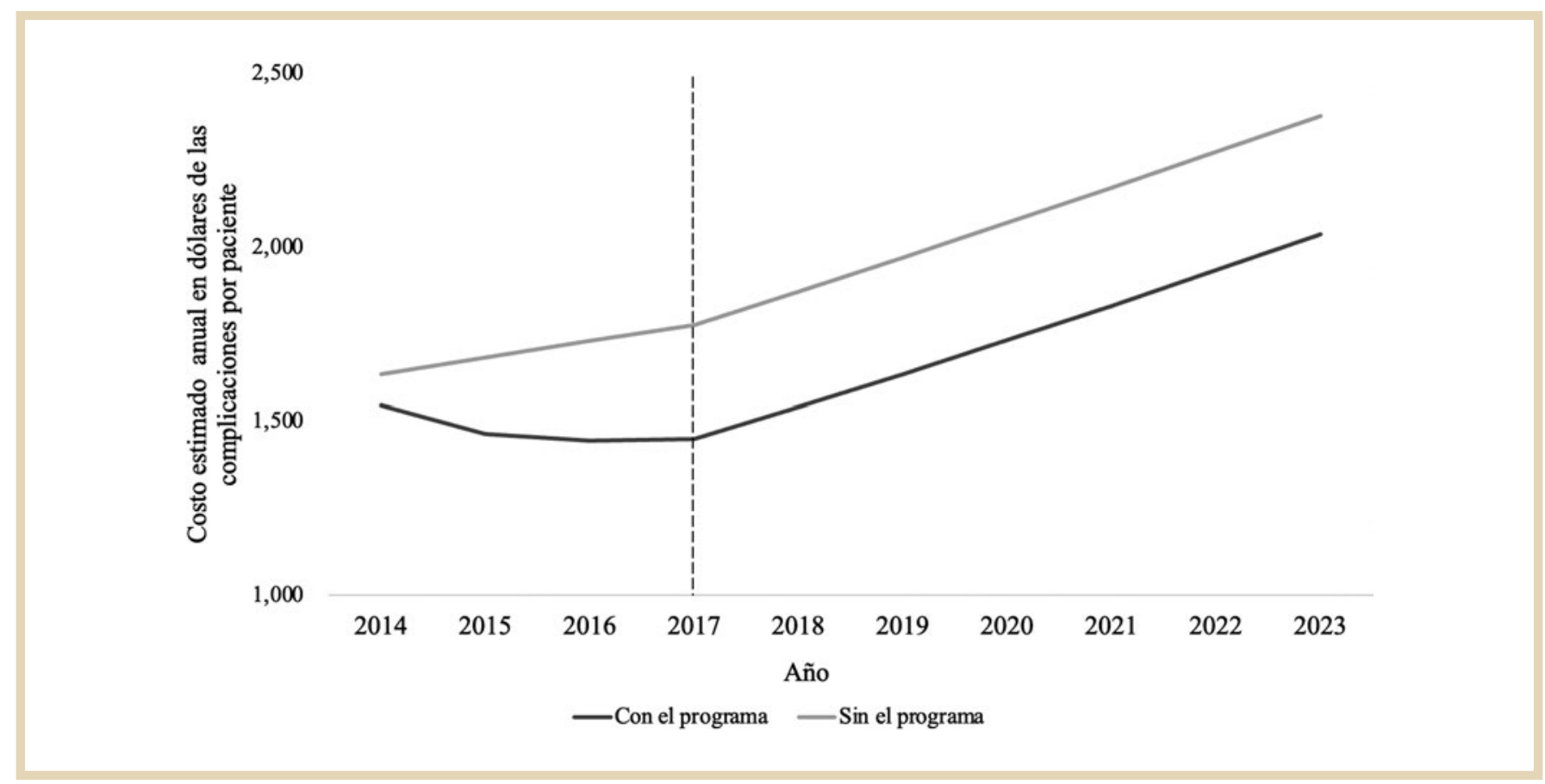

Figura 4. Costo estimado anual en dólares de las complicaciones por paciente.

TABLA 7. Costo estimado anual en dólares de las complicaciones por paciente y ahorros estimados

\begin{tabular}{|c|c|c|c|c|}
\hline & 2014 & 2015 & $\mathbf{2 0 1 6}$ & $\mathbf{2 0 1 7}$ \\
\hline $\begin{array}{c}\text { Costo por paciente } \\
\text { en la intervención }\end{array}$ & 1,546 & 1,465 & 1,445 & 1,450 \\
\hline $\begin{array}{c}\text { Costo por paciente } \\
\text { sin el PA }\end{array}$ & 1,636 & 1,683 & 1,730 & 1,776 \\
\hline Ahorros estimados & 90 & 218 & 285 & 326 \\
\hline
\end{tabular}

PA: programa automonitoreo.

como control glucémico ( $\mathrm{HbA1c}<7 \%$ ). Siendo la reducción promedio absoluta de HbA1c para los pacientes que redujeron sus niveles del $1.3 \%$, es de importancia mencionar que el análisis no permitió desagregar el beneficio individual de los componentes del programa, permitiendo un sesgo derivado del tratamiento farmacológico administrado de forma regular por los médicos de la CSS. Contrastando estos resultados con el aumento de $\mathrm{HbA} 1 \mathrm{c}$ esperado como consecuencia de la progresión natural de la enfermedad ( $+0.2 \%$ en nivel de HbA1c anual) establecido en la literatura ${ }^{13,24}$, podemos concluir que hay una mejora en al índice glucémico asociada a la intervención. Congruente con la literatura, nuestro análisis mostró diferencias significativas en los niveles de HbA1c dependiente del sexo del paciente ${ }^{26,27}$. Sin embargo, es interesante notar que la proporción de mujeres que mostraron mejoría es mayor que la de hombres; estos resultados entran en conflicto con los reportados en la literatura, que establecen que en pacientes de EE.UU. ${ }^{28}$ y Jordania ${ }^{29}$ los hombres y las mujeres tienen patrones similares de adherencia al automonitoreo. Esta discrepancia se debe al diseño experimental y a que las poblaciones de los estudios tienen diferencias étnicas y culturales importantes. Como resultado de la búsqueda realizada no obtuvimos información respecto a los patrones de adherencia al automonitoreo en países latinoamericanos, sin embargo, un estudio en Trinidad encontró, en cambio, que las mujeres siguen mejor el régimen de tratamiento, las dietas y asisten más a la clínica30.

Las limitaciones de nuestro estudio incluyen la falta de disponibilidad de variables demográficas 
asociadas al diagnóstico, tales como edad y raza ${ }^{31}$. El diseño experimental podría mejorarse, por ejemplo, vinculando los datos recabados por las navegadoras de salud con el historial clínico, administrado por los médicos, haciendo la asistencia a las citas con las navegadoras obligatorias y recopilando información clínica adicional como los niveles de HbA1c. Una debilidad del estudio es el hecho de que solo se recabaron registros del 56\% de los glucómetros repartidos, así como la ausencia de categorías específicas para diabetes autoinmune, diabetes monogénica, entre otras, las cuales fueron consideradas como parte de DM1. Para estimar el impacto económico se supuso que, aunque no haya datos recabados de los 35 mil pacientes, todos ellos usaron el glucómetro.

Aparte, está claro que la incidencia de las complicaciones está relacionada con los niveles de HbA1c, por lo que una reducción del $1 \%$ de $\mathrm{HbA} 1 c$ está asociada con una disminución relativa del $13-30 \%$ en el riesgo de las complicaciones ${ }^{5,6}$. Por esta razón, al implementar intervenciones de este tipo se puede producir un ahorro significativo en el gasto económico relacionado con la DM.

\section{CONCLUSIONES}

El empoderamiento de los pacientes por medio del entrenamiento en el uso e interpretación del glucómetro, sumado al acompañamiento de las navegadoras de salud y el cuidado médico que provee la CSS de Panamá probó beneficiar al mantenimiento del control glucémico.

Nuestros resultados motivan la discusión del impacto clínico y económico posible al impartir una intervención similar a una mayor población cubierta por la CSS. Adicionalmente, sería interesante implementar estas intervenciones en otros lugares de
Latinoamérica y analizar su impacto, en relación con las diferentes poblaciones. Proponemos, para trabajo futuro, el establecimiento de una motivación para incrementar la asistencia de los pacientes a las consultas con las navegadoras con el fin de recabar más datos y poder adaptar los consejos al progreso de cada paciente. Por ejemplo, se podría vincular la compra de insumos para el automonitoreo de los pacientes a la prestación de servicios médicos e historia clínica.

\section{AGRADECIMIENTOS}

Al personal directivo de las distintas direcciones ejecutivas nacionales, administrativo, médico, enfermería y multidisciplinario de la CSS que colaboraron en la creación e impulsaron las 32 clínicas de diabetes. Al personal gerencial, administrativo, nutricionistas y equipo técnico del consorcio AstorRoche, quienes hicieron una extraordinaria labor durante este proyecto; por último, al personal docente de la Iniciativa Latina del Joslin Diabetes Center por sus aportes. A nuestros pacientes, a nuestros familiares, a todos los Médicos de las Clínicas de Diabetes, a enfermería y equipo multidisciplinario de la Caja de Seguro Social que colaboraron en la creación, impulsaron y creyeron en las 32 clínicas de Diabetes. A todo el personal gerencial, administrativo, nutricionistas y equipo técnicodel Consorcio ASTOR-ROCHE por su labor extraordinaria. A la Universidad Latina de Panamá y a la Iniciativa Latina de Joslin Diabetes Center, Boston M A. por sus aportes.

\section{CONFLICTO DE INTERESES}

Los autores declaran no tener conflictos de intereses y cumplir con los códigos éticos de sus respectivas instituciones. 


\section{FINANCIAMIENTO}

El desarrollo del presente estudio no requirió de apoyo económico, de equipo o de otro tipo adicional a los recursos ya invertidos por la CSS de Panamá. El modelo utilizado para llevar a cabo el análisis fue financiado por Roche Servicios S.A.

\section{RESPONSABILIDADES ÉTICAS}

Protección de personas y animales. Los autores declaran que para esta investigación no se han realizado experimentos en seres humanos ni en animales.

\section{Confidencialidad de los datos. Los autores declaran} que han seguido los protocolos de su centro de trabajo sobre la publicación de datos de pacientes.

\section{Derecho a la privacidad y consentimiento informado.} Los autores declaran que en este artículo no aparecen datos de pacientes.

\section{BIBLIOGRAFÍA}

1. Diabetes overview. The path to understanding diabetes starts here [Internet]. American Diabetes Association [acceso: 26 de junio de 2020]. Disponible en: https://www.diabetes.org/diabetes

2. Papatheodorou K, Banach M, Bekiari E, Rizzo M, Edmond M. Complications of diabetes 2017. J Diabetes Res. 2018;3086167.

3. Barcelo A, Arredondo A, Gordillo Tobar A, Segovia J, Quiang A. The cost of diabetes in Latin America and the Caribbean in 2015: Evidence for decision and policy makers. J Glob Health. 2017;7(2):020410.

4. Ghavam S, Ahmadi MRH, Panah AD, Kazeminezhad B. Evaluation of $\mathrm{HbA}_{1} \mathrm{C}$ and serum levels of vitamin $\mathrm{D}$ in diabetic patients. J Family Med Prim Care. 2018;7(6):1314-8.

5. Stratton IM, Adler AI, Neil HA, Matthews DR, Manley SE, Cull CA, et al. Association of glycaemia with macrovascular and microvascular complications of type 2 diabetes (UKPDS 35): prospective observational study. BMJ. 2000;321:405-12.

6. Barraza-Lloréns M, Guajardo-Barrón V, Picó J, García R, Hernández C, Mora $\mathrm{F}$, et al. Carga económica de la diabetes mellitus en México, 2013. Ciudad de México, México: Funsalud; 2015.

7. Rosas-Guzmán J, Martinez-Sibaja C. Manual de automonitoreo glucémico: Documento de posición de la ALAD. Rev ALAD. 2019;9(Supl.1):103-15.

8. Nisar M, Asad A, Wagas A, Ali N, Nisar A, Qayyum M, et al. Association of diabetic neuropathy with duration of type 2 diabetes and glycemic control. Cureus. 2015;7(8):e302.

9. Shera AS, Jawad F, Magsood A, Jamal S, Azfar M, Ahmed U. Prevalence of chronic complications and associated factors in type 2 diabetes. J Pak Med Assoc. 2004;54(2):54-9.

10. Ahn JH, Jung SI, Yim SU, Kim SW, Hwang EC, Kwon DD. Impact of glycemic control and metformin use on the recurrence and progression of non-muscle invasive bladder cancer in patients with diabetes mellitus. J Korean Med Sci. 2016;31(9):1464-71.

11. American Diabetes Association. 2. Classification and diagnosis of diabetes: Standards of medical care in diabetes-2019. Diabetes Care. 2019;42(Suppl 1):S13-S28.
12. Qaseem A, Wilt TJ, Kansagara D, Horwitch C, Barry MJ, Forciea MA Hemoglobin A1c targets for glycemic control with pharmacologic therapy for nonpregnant adults with type 2 diabetes mellitus: A guidance statement update from the American College of Physicians. Ann Intern Med. 2018;168:569-76.

13. Sachiko O, Deshpandeac A, Yokomochi H, Takahashi O, Fukui T, Yamagata Z. HbA1c monitoring interval in patients on treatment for stable type 2 diabetes. A ten-year retrospective, open cohort study. Diabetes Res Clin Pract. 2017;135:166-71.

14. Brajkovich I, Aschner P, Taboada L, Camperos P, Gómez-Pérez R, Aure $G$, et al. Consenso ALAD.Tratamiento del paciente con diabetes mellitus tipo 2 y obesidad. Rev ALAD. 2019;9(Supl.1):40-75.

15. Sherwani SI, Khan HA, Ekhzaimy A, Masood A, Sakharkar MK. Significance of $\mathrm{HbA} 1 \mathrm{c}$ test in diagnosis and prognosis of diabetic patients. Biomark Insights. 2016;11:95-104.

16. Powers MA, Bardsley J, Cypress M, Duker P, Funnell MM, Fischl AH, et al. Diabetes self-management education and support in type 2 diabetes: A joint position statement of the American Diabetes Association, the American Association of Diabetes Educators, and the Academy of Nutrition and Dietetics. Clin Diabetes. 2015;34(2):70-80.

17. Liang X, Wang Q, Yang X, Cao J, Chen J, Mo X, et al. Effect of mobile phone intervention for diabetes on glycemic control: a meta-analysis. Diabet Med. 2011;28:455-63.

18. Perfiles de los países para la diabetes, 2016: Panamá [Internet]. Organización Mundial de la Salud [acceso: 26 de junio de 2020]. Disponible en: https://www.who.int/diabetes/country-profiles/pan_es.pdf?ua=1

19. Boletín 16. Estimaciones y proyecciones de la población en la República de Panamá, por provincia, comarca indígena y distrito, según sexo y edad: años 2000-2015 y 2020 [Internet]. Panamá: Instituto Nacional de Estadística y Censo. Disponible en: https://www.inec.gob.pa/publicaciones/Default3.aspx?ID_PUBLICACION=556\&ID_CATEGO$\mathrm{RIA}=3 \&$ ID SUBCATEGORIA $=10$

20. Prevalencia de factores de riesgo asociados a enfermedad cardiovascular (PREFREC) [Internet]. Gorgas [acceso: 26 de junio de 2020]. Disponible en: http://www.gorgas.gob.pa/PREFREC/CAPITULO\%209.pdf

21. Seguridad social en Centroamérica y República Dominicana: situación actual y desafíos. Washington, D.C.: Organización Panamericana de la Salud, Consejo de Instituciones de Seguridad Social de Centro América y República Dominicana; 2020.

22. Koga M, Suzuki S, Matsuo K, Tanahashi Y, Azuma H, Kasayama S. Calculation of $\mathrm{HbA} 1 \mathrm{c}$ and glycated albumin from serially measured self-monitored blood glucose in patients with type 1 diabetes mellitus. Clin Chim Acta. 2013;425:188-91.

23. Accu-Chek ${ }^{\circledR}$ [Internet]. Roche [acceso: 26 de junio de 2020]. Disponible en: https://www.accu-chek.com/us/glucose-monitoring/a1c -calculator.html

24. UK Prospective Diabetes Study Group. Intensive blood-glucose control with sulphonylureas or insulin compared with conventional treatment and risk of complications in patients with type 2 diabetes (UKPDS 33). Lancet. 1998;352:837-53.

25. GRD-IMSS: 2014. Arroyave-Loaiza M, Aburto-Mejía R, Dávila Torres J; IMSS. Grupos relacionados con el diagnóstico: Producto hospitalario. Instituto Mexicano del Seguro Social. Dirección de Prestaciones Médicas; 2016.

26. Saaddine JB, Fagot-Campagna A, Rolka D, Narayam KM, Geiss L, Eberhardt $M$, et al. Distribution of $\mathrm{HbA}(1 \mathrm{c})$ levels for children and young adults in the U.S.: Third National Health and Nutrition Examination Survey. Diabetes Care. 2002;2:1326-30.

27. Nakagami T, Oya J, Kasahara T, Uchigata Y. Effect of hemoglobin levels and sex on HbA1c levels among Japanese population. Diabetes and Endocrinology. 2017;1(1):3.

28. Fitzgerald JT, Anderson RM, Davis WK. Gender differences in diabetes attitudes and adherence. Diabetes Educ. 1995;21(6):523-9.

29. Abdulhameed A, AliAl-Hassan M, Sivarajan E. Self-efficacy, self-management, and glycemic control in adults with type 2 diabetes mellitus. Diabetes Complications. 2012;26(1):10-6.

30. Babwah F, Baksh S, Blake L, Cupid-Thuesday J, Hosein I, Sookhai A, et al. The role of gender in compliance and attendance at an outpatient clinic for type 2 diabetes mellitus in Trinidad. Rev Panam Salud Publica. 2006;19(2):79-84.

31. Herman WH, Cohen RM. Racial and ethnic differences in the relation- ship between $\mathrm{HbA} 1 \mathrm{C}$ and blood glucose: Implications for the diagno- sis of diabetes. J Clin Endocrinol Metab. 2012;(4):1067-72. 